\title{
Fractus, fracta, fractum. La indeterminación de la imagen y su relación con el acto narrativo en la era digital
}

El texto sobre la narración fractal, Fractal Narrative. About the Relationship Between Geometries and Technology and Its Impact on Narrative Spaces (2014) entra en su quinto año de vida, y, si bien en nuestro contexto tecnológico las trasformaciones sociales parecen escaparse de una obsoleta durée bergsoniana, algunos fenómenos expuestos en el texto siguen, a mi parecer, en continuo desarrollo. Recordando, por una parte, el proceso que me llevó a teorizar sobre la narración fractal y, por otra, el contexto tecnológico que me permitió evidenciar la naturaleza fractal de la narración audiovisual contemporánea, puedo constatar que, con la implementación del HTML5 y con la subsecuente asimilación de la multidimensionalidad del World Wide Web, el espacio fractal que diariamente habitamos y que hace cinco años se intuía inminente es hoy una realidad que se expande y abarca cada día más acciones humanas.

El concepto de la narración fractal nace de la constatación de dos teorías que progresivamente se consolidaron durante la segunda mitad del siglo pasado. La primera, siguiendo un orden cronológico, es la afirmación de Marshall McLuhan de que el medio es el mensaje. De hecho, esta tesis no solo transforma la comprensión de los medios, sino que también genera una subsiguiente "taxonomía térmica" de medios fríos y calientes, y su aplicación a las diferentes expresiones culturales. Esta frase ejemplifica y resume una condición humana bien precisa, que entiende al ser bajo una comprensión topológica, es decir, una forma que delinea una condición 
humana análoga al dasein heideggeriano ${ }^{1}$. De hecho, McLuhan no solo expuso de forma clara la relación entre el sujeto y el objeto, sino que, a través del vuelco generado por su frase, puso al sujeto en un espacio topológico donde la experiencia no es asimilada de forma egológica. El sujeto, para McLuhan, no representa una mera presencia en el espacio. Aceptando que el medio es el mensaje, se distingue la naturaleza topológica de la existencia que había sido descrita magistralmente por la fenomenología - especialmente por Merleau-Ponty- algunos años antes del fundamental Understanding Media: The Extensions of Man. Si recordamos las palabras de Merleau-Ponty, "[...] nous sommes au monde, et non pas seulement dans le monde" (1945, p. 281), las analogías con el concepto mcluhaniano saltan a la vista, más si cabe si retomamos el análisis que McLuhan hace sobre las tecnologías eléctricas, en especial del telégrafo.

During the mechanical ages we had extended our bodies in space. Today, after more than a century of electric technology, we have extended our central nervous system itself in a global embrace, abolishing both space and time as far as our planet is concerned. Rapidly, we approach the final phase of the extensions of man-the technological simulation of consciousness, when the creative process of knowing will be collectively and corporately extended to the whole of human society, much as we have already extended our senses and our nerves by the various media (McLuhan, 1994, pp. 3-4).

Para McLuhan, el telégrafo materializa una ruptura profunda en el ser. Por un lado, gracias al telégrafo, McLuhan constata que en su contexto tecnológico — profundamente marcado por la electricidad - la tecnología deja de ser una simple extensión del cuerpo: el telégrafo ejerce una verdadera externalización del sistema nervioso central. Por otro, se genera un colapso — de dimensiones aún desconocidas — de las precedentes y claramente definidas esferas de lo público y de lo privado, fenómeno sobre el que nos advierte Antonio Caronia con las siguientes palabras:

Nell'era dei mass media audiovisivi l'informazione sembra zampillare dall'interno della casa, dal telefono, dalla radio, dal televisore, dal computer. Il progressivo "addosmeticamento" dei media e la graduale

1 Sobre el dasein heideggeriano y sus analogías con las teorías de McLuhan y, en general, con los estudios de medios, véase Battin y Duarte (2018). 
"mediatizzazione" della casa sono avvenuti per adattamento reciproco.

Perciò se l'esterno, cioè il mondo, si rovescia dentro la casa nella forma di flussi informativi, l'interno, cioè la casa, può estroflettersi nel mondo (1996, p. 90).

Usando como instrumento de análisis la frase "el medio es el mensaje", a través del cual Caronia encauzó las teorías expuestas en el texto apenas mencionado, podemos ver que la grave invasión de la esfera privada - materializada con la presencia física en las salas de cada casa del hoy arcaico televisor- parece casi innocua en comparación con la gravísima extrapolación de lo privado a lo público.

Externalizando su sistema nervioso central, el sujeto creó una red infinita que se propagó y, a su vez, lo propagó por ella. El sujeto puso sus más íntimos deseos en la esfera pública, transformándose así en sujeto del poder. De hecho, sabemos que el sueño del poder siempre ha sido invadir la esfera privada, que hoy en día se convirtió en cosa pública; la esfera privada se publicó, o, como diría Flusser, se expuso, se exhibió, se prostituyó (2008). La presencia de la imagen del poder en la esfera privada, encarnada por el retrato del monarca, y el sueño de vigilancia constante en la reclusión del núcleo privado siempre fueron el deseo del poder. Como afirma Foucault en su fundamental Surveiller et punir, el sueño del poder era el de instaurar una suerte de toque de queda perene (1975). Es interesante resaltar que el dispositivo que, de cierta forma, le permite a Foucault concretizar la figura de la sociedad disciplinaria es el panóptico de Bentham. Cierto es que este tipo de construcción arquitectónica, que encarna el Zeitgeist del incipiente siglo de los medios de reproducción mecánica y del consumo bulímico de la imagen, fue concebida y destinada para ejercer un estricto control penitenciario, y, en consecuencia, ejemplifica el tipo de sociedad disciplinaria sobre el que teorizó Foucault ${ }^{2}$. Sin embargo, el interés de Foucault por el diseño arquitectónico de Bentham se concentraba en la organización del espacio sobre el que esta actúa. Es decir, partiendo del hecho en que el panóptico disloca la natural relación ver-ser visto, Foucault nos muestra claramente que el espacio social se expresa a través de relaciones entre objetos y sujetos y, en consecuencia,

2 Es interesante recordar que, para Bentham, este tipo de construcción representaría una sociedad completamente racionalizada y "correcta". En palabras de Bentham: "Morals reformedhealth preserved-industry invigorated-instruction diffused-public burden lightened [...] all by a simple idea in architecture" (Bentham, 1843, p. 39). 
el espacio social es un espacio topológico regido por un dispositivo que incluye todas las cosas, lingüísticas o no lingüísticas. De hecho, el dispositivo, como es puesto en evidencia por Agamben (2006), es la red que establece las relaciones de poder entre todos los elementos que forman el espacio social y, por esta razón, el dispositivo lo engloba todo, abarca lo dicho y no lo no dicho.

De la complejidad de este dispositivo, que no solo nos permite aflorar los tortuosos mecanismos sociales que sostienen una sociedad disciplinaria, sino también el concepto de dispositivo - y especialmente en el panoptismo como organización espacial y a su vez expresión de una sociedad disciplinaria-, emergen, a mi parecer, dos grandes deseos que han marcado profundamente nuestra especie. En efecto, en el concepto de la sociedad disciplinaria, así como es expuesto por Foucault, se revela la convergencia entre la necesidad de creación de un espacio capaz de contener y almacenar la información producida por la humanidad — que, como podemos imaginar, es infinito-y el deseo de orden, censura y transformación de este espacio y su contenido infinito. En otras palabras, por un lado, el producir información y, por otro lado, disponer de esta como instrumento de control social ${ }^{3}$.

Sin embargo, el concepto de narración fractal no nace de la simple constatación de que el contexto tecnológico influye directamente sobre el almacenamiento, el acceso y la distribución de la información. Siendo un firme discípulo de McLuhan, entiendo que su frase fundamental, "el medio es el mensaje”, posibilita la comprensión del papel de la tecnología en la construcción de las formas narrativas. Cierto es también que, como apenas expuesto en las líneas anteriores, el papel de la tecnología en la construcción y organización de las narraciones pasa a un segundo plano o, mejor, es producto de un proceso aún más profundo que viene a inserirse en el núcleo de la condición humana. El medio es el mensaje, porque es el medio quien configura y genera el espacio social. El medio es el mensaje, porque, como afirma Benjamin, la actividad cognitiva se transforma en contextos tecnológicos diferentes (Benjamin, 2002, p. 356). El medio es el mensaje, porque de este deriva nuestra percepción del

3 Es importante recordar que entendemos aquí información en su concepto etimológico de informare, es decir, la producción de formas mentales, imponer forma al evento o, en el contexto de la comunicación de masa, dar forma a la esfera pública. Como nota Roland Barthes con respecto a la multiplicación de las imprentas durante la revolución de 1789, la escritura en ese contexto mediático deja de comunicar o expresar para empezar a infligir, a forzar; incluso empieza a ir más allá del mismo lenguaje. En este punto, véase Barthes (2014). 
espacio y del tiempo, y, por ende, nuestra forma de relacionarnos con los objetos y sujetos que forman dicho espacio. El medio es el mensaje, porque el contenido es irrelevante, a diferencia de la forma de producirlo, de la velocidad de generarlo y de la forma de consumirlo. Más que producto de una tecnología, las formas narrativas son la más sincera expresión de un espacio experimentado, un espacio y un tiempo sentido, un espacio habitado y compartido de forma colectiva. En otras palabras, la forma narrativa es el intento fallido de legar una experiencia (Erlebnis), que a su vez es vivida a través de una tecnología y que infructuosamente es legada por la misma tecnología ${ }^{4}$.

El concepto de la narración fractal nace de esta constatación y se desarrolla sobre el terreno fértil que los estudios fundamentales de Gilles Deleuze prepararon durante la década de los ochenta. Es gracias a los trabajos de Deleuze, sobre todo Cinéma 1. L'Image-mouvement y Cinéma 2. L'Image-temps, que el análisis de la narración audiovisual tomó una distancia decisiva con los procesos de la lingüística. La ferviente búsqueda del esperanto visual, inuce en las primeras teorías del montaje de Eisenstein ${ }^{5}$, empieza a menguar decisivamente con los grandes aportes de Deleuze. Producto del ingreso a una fase postestructuralista, y, a mi juicio, también resultado directo de la inminente introducción de la tecnología digital en diversos campos sociales, las teorías de Deleuze sobre la narración audiovisual trajeron a un primer plano su naturaleza multidimensional, y sobre todo el carácter puramente espacial del acto narrativo en el campo audiovisual. Comunicar a través de la imagen en movimiento es, en otras palabras, organizar un espacio que será experimentado (erlebt) por el espectador. Hoy, los más recientes estudios que de forma multidisciplinar han conjugado la neurociencia y el cine demuestran que el espacio de la narración es realmente experimentado, es realmente vivido por el espectador ${ }^{6}$. Esos estudios, que empiezan a revelar el misterio de la creación de sentido a través de la imagen en movimiento, hoy le dan toda la razón a Deleuze: la narración audiovisual es una pura organización del espacio, ergo, una geometría.

Durante la década de los ochenta, cuando Deleuze publicó los dos textos sobre el cine, el carácter espacial de la narración audiovisual empezaba a ser evidente. La inminente tecnología digital empezaba 
a diseñar una serie de construcciones hipertextuales que ponían al desnudo el error del estructuralismo: la narración a través de la imagen en movimiento no puede ser asimilada como una lengua:

\begin{abstract}
La référence au modèle linguistique finit toujours par montrer que le cinéma est autre chose, et que, si c'est un langage, c'est un langage analogique ou de modulation. On peut dès lors croire que la référence au modèle linguistique est un détour dont il est souhaitable de se passer
\end{abstract} (Deleuze, 1990, p. 76).

Es precisamente sobre la posibilidad de construir narraciones de carácter hipertextual en el campo audiovisual que el concepto de la narración fractal empezó a tomar forma. Por una parte, las estructuras hipertextuales empezaban a reflejar el proceso mental de vincular información, de conectar lugares. Como ya notaba Vannebar Bush (1945), describiendo a Memex en el breve ensayo de su autoría, existen claras analogías entre el proceso mental y la construcción de una red hipertextual electrónica. Memex representa el verdadero primer paso hacia la materialización de la estructura hipertextual, y, en consecuencia, esta nueva forma de almacenar, generar, asociar y organizar la información acercaba progresivamente el acto narrativo a una pura organización del espacio y su fruición a una simple navegación del espacio precedentemente establecido.

Todo indicaría que después del desarrollo de la red telegráfica, la verdadera externalización de nuestro sistema nervioso central, la organización de la información ha empezado a organizarse de forma hipertextual. Es curioso notar que la forma hipertextual ha sido puesta en evidencia también en contextos tecnológicos precedentes a la electrónica. Como lo recuerdan Genette (1982), Roland Barthes (2014) o Jay David Bolter (1991, p. 22), quien describe la forma hipertextual como el eco que existe entre palabras, afirmando así que cada palabra, párrafo o texto evoca uno precedente, $y$, en consecuencia, la forma hipertextual existe de forma clara en el texto impreso. Todo texto tiene relación con uno precedente, y es por esto que Bolter entiende la escritura como la creación de una network de elementos verbales en los que la jerarquía juega un papel principal tanto en la estructura espacial como en el orden y proceso del raciocinio. Sin embargo, es curioso constatar que, a pesar de que la percepción de la escritura como tejido hipertextual sea una comprensión con amplio consenso, es precisamente en el seno de la informática —es 
decir, durante la segunda mitad del siglo pasado- donde emerge y se desarrolla a cabalidad el concepto de hipertexto. De hecho, el vocablo fue presentado por Ted Nelson en la década de los sesenta.

Creo fundamental recordar que la palabra hipertexto se basa sobre la exclusiva naturaleza multimedia del medio digital, naturaleza que se vislumbraba desde la tecnología video-electrónica, tecnología intermedia entre lo analógico y lo digital ${ }^{7}$. Si leemos con detenimiento A file Structure for The Complex, The Changing and The Indeterminate de Ted Nelson, podemos ver claramente que la estructura del concepto de hipertexto se posiciona en el campo semántico de un forma textual flexible y multitextual que, por su naturaleza caótica, impone la ausencia total de soporte material, y, en consecuencia, impone un nuevo orden que acepta — como un complexus (Morin, 2005) — una serie de factores heterogéneos, pero que en su orden particular no aplica una fuerza de homogenización. Recordemos las palabras de Nelson describiendo su concepto:

Let me introduce the Word "Hypertext" to mean a body of written or pictorial material interconnected in such a complex way that it could not conveniently be presented or represented on paper. It may contain summaries, or maps of its contents and their interrelations: it may contain annotations, additions and footnotes from scholars who have examined it. Let me suggest such an object and system, properly designed and administrated, could have great potential for education, increasing the student's range of choices, his sense of freedom, his motivation, and his intellectual grasp. Such a system could grow indefinitely, gradually including more and more of the world's written knowledge (Nelson, 1965, p. 42).

El concepto de hipertexto, como lo define Ted Nelson, comprende a mi parecer los fenómenos narrativos y sociales que se han desarrollado y consolidado a través de los prefijos más usados y discutidos en los estudios sociales y mediáticos en los últimos años: trans, inter, cross. ¿No es el fenómeno intermedia de Dick Higgins un fenómeno que pone la práctica artística en el contexto hipertextual ${ }^{8}$ ? ¿No engloba la estructura

7 A este propósito, véase Spielmann (2005).

8 Cabe recordar que, para Higgins, el acercamiento intermedial del artista también deriva de un cambio de sensibilidad generado por la sobreposición de medios, algo que notamos también en el concepto de hipertexto de Nelson. 
hipertextual teorizada por Nelson las principales características de la narración transmedia como definida sucesivamente por Jenkins, entre ellas la no linealidad de la narración y su apertura? Si el hipertexto implica la ausencia de soporte, y, en consecuencia, actúa una fuerza de desmaterialización del medio, ¿no es un efecto natural de la hipertextualidad la convergencia mediática, y a su vez la narración transmedial propuesta por Jenkins? ¿No es el paso de un medio a otro el simple producto de la adquisición de una organización hipertextual que no concibe el fenómeno como un paso, mas como el uso natural de la misma forma hipertextual ${ }^{9}$ ? De hecho, el concepto de hipertextualidad, como resalta Robert Coover, indica un espacio narrativo multidimensional y posiblemente infinito, ya que este presenta una serie infinita de posibles vínculos, redes, networks, que pueden ser programados o simplemente aleatorios ${ }^{10}$. Como podemos ver, el carácter multidimensional del hipertexto implica la lógica intermedia puesta en evidencia por Higgins, es decir, el uso dialéctico entre los diferentes medios, tal y como él mismo sostiene:

For the last ten years or so, artists have changed their media to suit this situation, to the point where the media have broken down in their traditional forms, and have become merely puristic points of reference. The idea has risen, as if by spontaneous combustion throughout the entire world, that these points are arbitrary and only useful as critical tools, in saying that such-and-such a work is basically musical, but also poetry. This is the intermedial approach, to emphasize the dialectic between the media. A composer is a dead man unless he composes for all the media and for his world (Higgins, 1967).

Pero, a su vez, la multidimensionalidad apenas mencionada también implica la lógica transmedia propuesta por Jenkins, ya que la forma multidimensional del hipertexto implica la multiplicación al infinito de diferentes puntos de entrada y de salida de la estructura narrativa. Esto no solo indica que la narración se libera definitivamente de la linealidad -ya postulada por Aristóteles_, sino también que la narración adquiere dimensiones tan grandes que no puede ser "contenida" en un solo

\footnotetext{
9 Véase Landow (2006).

10 A este propósito, consúltese Duarte (2014), en especial, el capítulo "Hypertext: A digital Episteme".
} 
medio. En palabras de Jenkins, en referencia a su concepto de transmedia storytelling:

The Matrix is entertainment for the age of media convergence, integrating multiple texts to create a narrative so large that it cannot be contained within a single medium. The Wachowski brothers played the transmedia game very well, putting out the original film first to stimulate interest, offering up a few Web comics to sustain the hard-core fan's hunger for more information, launching the anime in anticipation of the second film, releasing the computer game alongside it to surf the publicity, bringing the whole cycle to a conclusion with The Matrix Revolutions, and then turning the whole mythology over to the players of the massively multiplayer online game. Each step along the way built on what has come before, while offering new points of entry (Jenkins, 2008, p. 95).

La multidimensionalidad también abarca el concepto crossmedia, que reconoce al Internet como el espacio donde los nodos narrativos se desarrollan. De hecho, la multidimensionalidad del hipertexto es producto de un fenómeno progresivo de fragmentación de la forma tradicional de los medios de comunicación, una fragmentación que se inició desde la popularización de las tecnologías eléctricas y que, durante la segunda mitad del siglo pasado, alteró profundamente la difusión, el consumo y la producción de las expresiones culturales.

La serie inter, trans, cross, en mi opinión, no es otra cosa que el intento de conceptualizar la progresiva hipertextualización de la narración, y, al mismo tiempo, la constatación de que el artista enfrentaba la necesidad, desde la década de los cincuenta, de desarrollar nuevos mecanismos de creación de sentido, esta vez capaces de apropiarse de los mecanismos de medios precedentes, ora obsoletos. Este fenómeno, que lógicamente atañe a todas las expresiones culturales, se materializaba en la desintegración gradual de los medios tradicionales a favor de las plataformas multimedia y multifuncionales, donde los objetos culturales, hechos precedentemente para otro medio especifico, empezaban a converger ${ }^{11}$. Claramente, estos complejos fenómenos de remediación ${ }^{12}$ que se entrelazaban sin duda con

\footnotetext{
11 A este propósito, véase Casetti (2011). También aconsejo sobre los fenómenos transmediales y de adaptación Deprêtre y Duarte (2019).

12 Sobre el concepto de remediación, véase Bolter y Grusin (2000).
} 
una sociedad compleja y fuertemente influenciada por el capitalismo que no es más que una fuerza de producción de sentido, y cuya fábrica de deseos se materializaba en Hollywood-eran el producto de la irrupción de una nueva tecnología. En el caso del concepto de intermedia, era la tecnología videoelectrónica que, iniciando a desmaterializar el medio, imponía una relación dialéctica entre medios capaces de articularse. En el caso del transmedia, era el vislumbre del espacio infinito de Internet, donde se ejercía la apertura de la obra; esta vez, a diferencia del fenómeno analizado por Eco (2006) su apertura no era una simple alusión. Internet abría la obra, y esta apertura tomaba cuerpo a través de las primeras experiencias del $\mathrm{P} 2 \mathrm{P}$.

Como fue expuesto en las líneas precedentes, por una parte, la afirmación de McLuhan permitía una comprensión diferente sobre la estrecha relación entre la tecnología y las formas narrativas. Por otra, estas formas narrativas, en la obra de Deleuze, habían adquirido una comprensión puramente espacial. El acto narrativo pasaba así a ser pertinencia de la geometría y no de la lingüística; se alejaba de la semiótica y se arraigaba en la fenomenología. El raciocinio que se formaba requería el trazo de un paralelo que permitiera no solo el examen de la estrecha relación entre tecnología, geometría, procesos sociales, construcción de imaginario colectivo y Raumgestaltung, sino que a su vez permitiera identificar la geometría que, conjugándose con la tecnología digital, estuviera influenciando la construcción de los espacios narrativos de nuestros días. Es de esta forma que la geometría fractal empezó a poblar los análisis de las nuevas tendencias narrativas, sobre todo, las experiencias interactivas, así como aquellas que se generaban gracias a la práctica P2P.

Los principios de la geometría fractal — desarrollada exclusivamente por la capacidad de cálculo que ofrecen los computadores- empezaban a converger con algunos fenómenos narrativos, que también eran exclusivos de la tecnología digital. La geometría fractal, de hecho, había conferido forma al infinito, había hecho converger el infinito potencial y el infinito actual en maravillosas figuras autosimilares. Los fractales son espacios infinitos capaces de autogenerarse por su estructura abierta. Como en una página web, capaz de aceptar siempre más información (como es el caso de YouTube), los fractales representan espacios abiertos y recursivos capaces de almacenar cantidades infinitas de información.

Es también una característica de la geometría fractal proporcionar una imagen al caos, reconocer la irregularidad en la naturaleza, la cual 
representa la forma de las estructuras narrativas en nuestro contexto digital. En efecto, el acto narrativo - que como pudimos ver es una pura organización del espacio y que, a su vez, es un tipo de organización que deriva directamente del contexto tecnológico- genera, en nuestro contexto digital, estructuras narrativas carentes de inicio y de final. Estructuras narrativas que diluyen la visión aristotélica de la narración. Adicionalmente, en nuestro contexto digital, debido a la inmaterialidad y a la apertura fractal del espacio narrativo, la creación de la narración se transforma progresivamente en un acto colectivo, en el que la tecnología también desempeña un papel activo.

A mi juicio, son estos últimos fenómenos en el ámbito de la narración audiovisual los que se pondrán en un futuro próximo en el centro del debate. Se tendrá que llegar a cuentas con la influencia de la tecnología en la construcción de los espacios narrativos. Esta vez, no será, sin embargo, una influencia pasiva. Se deberá analizar una presencia activa de la tecnología en el proceso creativo. Habrá de entenderse que los espacios narrativos en el ámbito digital son formas fractales en continua y colectiva transformación, y que, por esta misma razón, se tendrán que replantear, hasta en términos jurídicos, conceptos tales como los de autoría, texto, soporte, difusión, etc. Sin embargo, estos temas, puestos en un plano antropocéntrico, no serán de gran relevancia por el simple hecho de enfrentarnos al fenómeno de la completa enajenación del ser humano del proceso creativo y de las formas expresivas, hasta hoy de su exclusiva potestad.

Creo que, en un futuro no muy lejano — viendo las recientes experiencias de edición exclusivamente realizadas por un software-, la producción de sentido, a través de la imagen en movimiento, empezará a ser tarea de la máquina. Esta posibilidad no parece ser descabellada si consideramos que el mundo empieza a ser exclusivamente poblado por personas que aprendieron a comunicar, a hablar, con un televisor y no con su madre. Parece ser una consecuencia natural de un proceso iniciado por la tecnología videoelectrónica ${ }^{13}$. La enajenación del componente humano podría también ser una consecuencia natural, si tenemos en cuenta la naturaleza indeterminada de la imagen en movimiento. Creo que la ausencia de una relación sólida entre el significante y el significado en el campo audiovisual hace de este un terreno más fértil para la

13 A este propósito, véase Goldsen (1977). 
experimentación y a su vez para la comprensión del papel de la tecnología digital, y de la inteligencia artificial si se quiere, en la expresión artística.

Es precisamente sobre estas reflexiones que se fundó la iniciativa de publicar un número monográfico acerca de la indeterminación de la imagen y de su relación con el acto narrativo en la era digital. Es en medio de este fenómeno donde auspicio que se dirigirá la atención de los estudios tanto sociales como artísticos. Aquí, en este texto monográfico, considero que son analizados los mayores fenómenos del tema apenas expuesto. En este número, el lector encontrará una serie de reflexiones sobre la relación entre la imagen en movimiento y el establecimiento de una red mundial de comunicación. Encontrará también las implicaciones estéticas, sociales y filosóficas que este fenómeno conlleva. El número adicionalmente goza de una interesante contribución sobre una práctica surrealista - práctica que, por cierto, se basa sobre la indeterminación de la imagen-y los límites que esta encontró en un contexto anterior al digital. El número tiene además el placer de presentar un trabajo en el que se analizan de forma perspicaz las interesantes formas narrativas que emergen del uso de la tecnología digital como instrumento de liberación de la opresión ejercida por la teocracia iraní. El uso de la tecnología digital se hace asimismo presente en una contribución que en su análisis abraza los fenómenos de fragmentación y de interacción con los espacios narrativos digitales. Esta contribución, que ahonda de manera exquisita la cuestión del tiempo diegético en un contexto de narraciones interactivas, introduce un breve ensayo que pone en evidencia, a través de la musicología - y, en consecuencia, de la expresión musical, arte máxima que se expresa a través del tiempo- la presencia de formas fractales en la música minimalista. El número no hubiera podido ignorar la complejidad social en la que estas manifestaciones artísticas se desarrollan. Es por eso que cierra la serie de ensayos con una interesante contribución que estudia la obra de Ruben Östlund, la crítica de lo contemporáneo y de las formas de reificación de las expresiones culturales. El texto es sin duda una contribución que abre las puertas a un replanteamiento de los fenómenos expuestos por la Escuela de Frankfurt —a través de un pertinente análisis de Baudrillard - al estudio de la producción de valor de una industria cultural en el medio de la revolución digital, en el medio de un mundo globalizado que encuentra en el modelo del iluminismo la forma de la opresión. Por último, sin que por ello sea menos importante, el número monográfico cierra con una reflexión estrictamente saldada al modelo del iluminismo apenas mencionado, y, en mi opinión, la organización arquitectónica que 
mejor lo ha representado: el museo, expresión máxima del colonialismo. Cerramos el número con una reseña sobre el texto emblemático de la museología, en la que, no solo se presenta el análisis del complejo expositivo, sino también se expone la analogía entre el museo como creación de una forma narrativa - a través de una Raumgestaltung bien precisa-y las narraciones digitales. De esta analogía emerge también un vector de análisis de la sociedad disciplinaria y su versión más actual, su versión inmaterial, su versión digital.

\section{Referencias}

Agambem, G. (2006). Che cos'è un dispositivo? Roma: Nottetempo.

Barthes, R. (2014). Le Degré Zéro de l'écriture. Paris: Seuil.

Battin, J. M. \& Duarte, G. A. (Eds.). (2018). We Need To Talk About Heidegger. Essays Situating Martin Heidegger In Contemporary Media Studies. Berlin: Peter Lang.

Bentham, J. (1843). The Works of Jeremy Bentham, vol. IV. J. Bowring (Ed.). London: William Tait.

Bolter, J. D., \& Grusin, R. A. (2000). Remediation: Understanding new media. Cambridge: міт Press.

Bush, V. (1945, julio). As We May Think. The Atlantic Magazine. Recuperado de http://www.theatlantic.com/magazine/archiv/1945/07/as-we-maythink/3881/\#

Caronia, A. (1996) Il corpo virtuale. Dal corpo robotizzato al corpo disseminato. Padova: Franco Muzzio Editore.

Casetti, F. (2011). Back to the Motherland: The Film Theatre in the Postmedia Age. Screen, 52(1), 1-12.

Casetti, F. (2015). La galassia Lumière. Sette parole chiave per il cinema che viene. Bompiani: Milano.

Deleuze G. (1990). Pourparlers, Paris: Minuit.

Deprêtre, E. \& Duarte, G. A. (Ed.). (2019). Transmédialité, bande dessinée Ė adaptation. Clermont-Ferrand: Presses Universitaires Blaise Pascal.

Duarte, G. (2014). Fractal Narrative. About the Relationship Between Geometries and Technology and Its Impact on Narrative Spaces. Bielefeld: Transcript.

Eco, U. (2006/1962). Opera aperta. Forma e indeterminazione nelle poetiche contemporanee. Milano: Bompiani.

Eisenstein, S. M. (1943). The Film Sense (editado y traducido por J. Leyda). London: Harcourt.

Eisenstein, S. M. (1949). Film Form: Essays on Film Theory (editado y traducido por J. Leyda). London: Harcourt.

Flusser, V. (2008). Kommunikologie weiter denken. Die Bochumer Vorlesungen. Frankfurt am Main: Fischer. 
Foucault, M. (1975). Surveiller et punir. Naissance de la prison. Saint-Amand:

Gallimar.

Gallese, V. \& Guerra, M. (2015). Lo schermo Empatico. Cinema e neuroscienze: Milano: Rafaello Cortina Editore.

Genette, G. (1982). Palimpsestes. La littérature au second degré. Saint-Amand: Gallimar.

Goldsen, R. (1977). The Show and Tell machine: How Television Works and Works You Over. New York: Dial Press.

Landow, G. P. (2006). Hypertext 3.0. Critical Theory and New Media in an Era of Globalization. Baltimore: The johns Hopkins University Press.

Jenkins, H. (2008). Convergence Culture. Where Old and New Media Collide. New York, NYU Press.

Higgins, D. (1967). Statement on Intermedia. En W. Vorstell (Ed.), Dé-coll/age (décollage). Frankfurt: Typors Verlag.

McLuhan, M. (1994). Understanding Media. The Extensions of Man. Cambridge: MIT Press.

Merleau-Ponty, M. (1945). Phénoménologies de la perception. Paris: Galimard.

Morin, E. (2005). Introduction à la pensée complexe. Paris: Points.

Nelson, T. (1965). A file Structure for the Complex, The Changing and The Indeterminate. Proceedings of the ACM 20th National Conference.

Spielmann, Y. (2005). Video. Das reflexive Medium. Frankfurt am Main: Suhrkamp.

German A. Duarte

Doctor en Filosofía con énfasis en Filosofía Cinematográfica de la Ruhr-Universität Bochum Profesor de historia del cine y teoría de medios de comunicación de Freie Universität Bozen-Bolzano, Italia https://orcid.org/0000-0002-0902-7790 gduarte@unibz.it DOI: $10.21789 / 25007807.1445$ 